\title{
OSTEONECROSIS DE CONDUCTO AUDITIVO EXTERNO SECUNDARIA A BIFOSFONATOS. REVISIÓN SISTEMÁTICA DE LA LITERATURA
}

\section{Osteonecrosis of the external auditory canal secondary to bisphosphonates. Systematic review}

\author{
Eugenia LÓPEZ-SIMÓN; Patricia CORRIOLS-NOVAL; Nathalia CASTILLO-LEDESMA; \\ Carmelo MORALES-ANGULO \\ Servicio de Otorrinolaringología. Hospital Universitario Marqués de Valdecilla (HUMV), Santander. España. \\ Correspondencia: eugenialopez@usal.es
}

Fecha de recepción: 24 de junio de 2019

Fecha de aceptación: 6 de agosto de 2019

Fecha de publicación: 8 de agosto de 2019

Fecha de publicación del fascículo: 1 de diciembre de 2019

Conflicto de intereses: Los autores declaran no tener conflictos de intereses

Imágenes: Los autores declaran haber obtenido las imágenes con el permiso de los pacientes

Política de derechos y autoarchivo: se permite el autoarchivo de la versión post-print (SHERPA/RoMEO)

Licencia CC BY-NC-ND. Licencia Creative Commons Atribución-NoComercial-SinDerivar 4.0 Internacional

Universidad de Salamanca. Su comercialización está sujeta al permiso del editor

\begin{abstract}
RESUMEN: Introducción y objetivo: La osteonecrosis benigna del conducto auditivo externo (CAE) es un efecto secundario bastante desconocido del tratamiento con bifosfonatos (BFS). Nuestro objetivo es describir la forma de presentación y el manejo terapéutico de los casos descritos en la literatura. Método: Se realizó una revisión sistemática de la literatura de los casos de osteonecrosis del CAE secundaria a BFS desde septiembre 2003 a febrero 2019. Se siguieron los criterios PRISMA evaluando las bases de datos PubMED. Resultados: Se han identificado 7 estudios que incluyen un total de 17 pacientes con osteonecrosis del CAE. El 76\% fueron mujeres y el 24\% restante varones, con edades comprendidas entre 51 y 89. El tiempo de evolución hasta la aparición de complicaciones osciló entre 15 meses y 20 años. La forma de presentación fue la úlcera sobreinfectada con exposición ósea unilateral en $41 \%$ de los casos. La zona de aparición inicial fue el suelo del CAE en el 100\%. Se realizó tomografía axial computarizada al 47\% de los casos observando signos de necrosis ósea; y dos biopsias con resultado de osteomielitis. 9 pacientes (53\%) fueron tratados con éxito con antibioterapia \pm corticoides tópicos, mientras que los 8 restantes (47\%) precisaron tratamiento quirúrgico además de la retirada de BFS. Discusión/Conclusiones: La osteonecrosis de CAE es un efecto secundario muy infrecuente del uso de BFS. La forma de presentación más común es la
\end{abstract}




\section{OSTEONECROSIS DE CONDUCTO AUDITIVO EXTERNO SECUNDARIA A BIFOSFONATOS. REVISIÓN SISTEMÁTICA DE LA LITERATURA \\ LÓPEZ-SIMÓN E ET AL}

úlcera indolora en el suelo del conducto. Para su tratamiento con frecuencia es suficiente con la limpieza local y administración de antibióticos tópicos.

Palabras clave: bifosfonatos; conducto auditivo externo; osteonecrosis; osteoporosis.

SUMMARY: Introduction and objective: Benign osteonecrosis of the external auditory canal (EAC) is a side effect pretty unknown due to bisphosphonate (BPs) therapy. Our aim is to describe the way of presentation and treatment of cases published in literature. Method: We did a systematic review of patients with diagnose of osteonecrosis of the external ear canal secondary to bisphosphonates from September 2003 till February 2019. We followed PRISMA criteria and evaluated PubMed database. Results: 7 articles were identified including a total of 17 patients with osteonecrosis of EAC. $76 \%$ were women and $24 \%$ men, within ages between 51 and 89 . The duration time till complications appeared ranged from 15 months to 20 years. The most common presentation was as a unilateral ulceration with bone exposure in $41 \%$ of cases. Floor of the EAC was the initial place in $100 \%$. Computed Tomography was performed in $47 \%$ of patients showing bone necrosis; and two biopsies with osteomyelitis signs. 9 patients (53\%) were successfully treated with antibiotics and topical corticosteroids, while 8 cases (47\%) needed surgical treatment in addition to the withdrawal of BFS. Discussion/Conclusions: Osteonecrosis of EAC is a rare side effect of BPs treatment. Painless ulceration on the EAC floor is the most common onset. Topical antibiotics and local debridement are frequently enough for complete healing.

Keywords: biphosphonates; external auditory canal; osteonecrosis; osteoporosis.

\section{INTRODUCCIÓN}

Lo osteoporosis es un problema muy común sobre todo en la población femenina relacionado con la edad, que da lugar a la aparición de fracturas óseas que provocan gran alteración de la calidad de vida de los pacientes que la padecen [1]. Los bifosfonatos (BFS) constituyen un grupo de medicamentos ampliamente utilizados en el tratamiento de la osteoporosis y diferentes tipos de cáncer $[2,3]$. Debido a su efecto inhibitorio en la reabsorción de hueso, han demostrado su capacidad de reducción del riesgo de lesiones esqueléticas en estos pacientes $[1,4]$.

La osteonecrosis es una condición benigna caracterizada por la exposición de hueso necrótico asociada generalmente al uso de BFS [5]. La osteonecrosis de mandíbula es un efecto secundario infrecuente pero muy conocido del tratamiento con BFS descrito inicialmente en el año 2003 por Khosla et al [6]. Sin embargo, la osteonecrosis del conducto auditivo externo (CAE) es un hallazgo raro y desconocido del tratamiento con estos fármacos. Su aparición es, mayoritariamente, en forma de úlcera indolora localizada en el suelo de la parte ósea del CAE bajo una capa de necrosis. En el año 2006, Polizzotto et al describieron el primer caso de osteonecrosis del CAE secundaria a dicha medicación y que ha sido corroborado por otros autores en diferentes estudios [7].

Nuestro objetivo principal es realizar una revisión sistemática de los casos de osteonecrosis del CAE asociados a consumo de bifosfonatos de forma oral o intravenosa publicados en la literatura médica, determinar cuáles son las características clínicas, su manejo diagnóstico y terapéutico.

\section{MATERIAL Y MÉTODO}

El método de análisis y los criterios de inclusión fueron especificados previamente y recogidos en forma de protocolo. La revisión se realizó siguiendo los criterios PRISMA (Preferred Reporting Items for Systematic Reviews and Meta-analysis) [16]. Se 


\section{OSTEONECROSIS DE CONDUCTO AUDITIVO EXTERNO SECUNDARIA A BIFOSFONATOS. REVISIÓN SISTEMÁTICA DE LA LITERATURA}

definió la osteonecrosis del conducto como el área de tejido óseo expuesto en el CAE en un paciente tratado con BFS en ausencia de tratamiento radioterápico previo en la región del hueso temporal o áreas próximas, que no cura durante un periodo de seguimiento de 8 semanas después del diagnóstico [5]. Esta definición se realizó en base a la descrita previamente para la osteonecrosis mandibular de la Asociación Americana de Cirujanos Orales y Maxilofaciales (AAOMS).

Los criterios de inclusión fueron: 1) Casos publicados de pacientes mayores de 18 años de cualquier sexo con diagnóstico de osteonecrosis del CAE en ausencia de tratamiento radioterápico previo. 2) Estudios que incluyesen una descripción clínica básica de la edad, sexo, presentación clínica, tipo de estudio radiológico realizado y tratamiento realizado. 3) Identificación del tipo de medicación y tiempo de tratamiento antes del inicio de la necrosis. 4) Cumpliese el criterio diagnóstico de osteonecrosis de CAE comentado previamente. 5) Estudios escritos en cualquier idioma.

Se excluyeron de la revisión: 1) Estudios de casos publicados de pacientes menores de 18 años que consumieron BFS como tratamiento de osteogénesis imperfecta. 2) Publicaciones con material redundante. 3) Estudios realizados en animales. 4) Pacientes sometidos a tratamiento radioterápico en el área maxilofacial. 5) Estudios en relación con otras entidades similares a la osteonecrosis del hueso mandibular o cuyo origen es atribuido a otros fármacos distintos de los bifosfonatos.

Se realizó una búsqueda activa de casos en la base de datos MEDLINE vía PubMed, desde septiembre 2003 (año de publicación del primer caso de osteonecrosis de mandíbula) a marzo de 2019 incluido. Además, se realizó una búsqueda manual por parte de los autores en diferentes revistas como Bone, British Journal of Oral and Maxillofacial Surgery, International Journal of Oral and Maxillofacial Surgery, Journal of Oral Maxillofacial Surgery, Journal of Cranio-Maxillofacial Surgery, Oral Surgery, Oral Medicine Oral Pathology and
Oral Radiology and Oral Oncology. Cualquier artículo potencialmente relevante relacionado con los autores o artículos obtenidos fue también evaluado.

Se usaron los siguientes términos para realizar la búsqueda de casos publicados en dichas bases de datos: ((diphosphonates OR bisphosphonates) AND (osteonecrosis OR external auditory canal osteonecrosis OR ear)). En la búsqueda se utilizaron términos MeSH y palabras libres. La búsqueda fue adaptada convenientemente para ser utilizada en las bases de datos Web Of Science y Biblioteca Cochrane.

La secuencia de selección de los artículos se resume en la Figura 1.

Dos autores realizaron de forma independiente la selección de artículos de forma estandarizada de acuerdo con los criterios previamente establecidos. Desacuerdos entre los dos autores fueron resueltos por consenso. Después del screening inicial, se investigó el texto completo de los artículos seleccionados. Las razones de exclusión de los artículos que fueron eliminados se registraron. Se extrajeron y analizaron los siguientes datos de todos los artículos incluidos en la revisión realizada: (1) características de los pacientes (incluyendo sexo, edad, enfermedad de base diagnosticada y factores asociados); (2) identificación del tipo de bifosfonato, vía de administración, dosis y duración del tratamiento; (3) síntomas presentes; (4) lugar de aparición de la osteonecrosis; (5) presencia/ausencia de sobreinfección; (6) realización de TC o RM; (7) tipo de tratamiento realizado y (8) aparición de recurrencia.

La calidad metodológica de los estudios incluidos y la posibilidad de sesgos fueron evaluados de forma independiente por los dos autores. En caso de discrepancia entre ambos, un tercer revisor actuó como mediador. Se consideró la posibilidad de sesgo de publicación y la falta de homogeneidad entre artículos. 


\section{OSTEONECROSIS DE CONDUCTO AUDITIVO EXTERNO SECUNDARIA A BIFOSFONATOS. REVISIÓN SISTEMÁTICA DE LA LITERATURA \\ LÓPEZ-SIMÓN E ET AL}

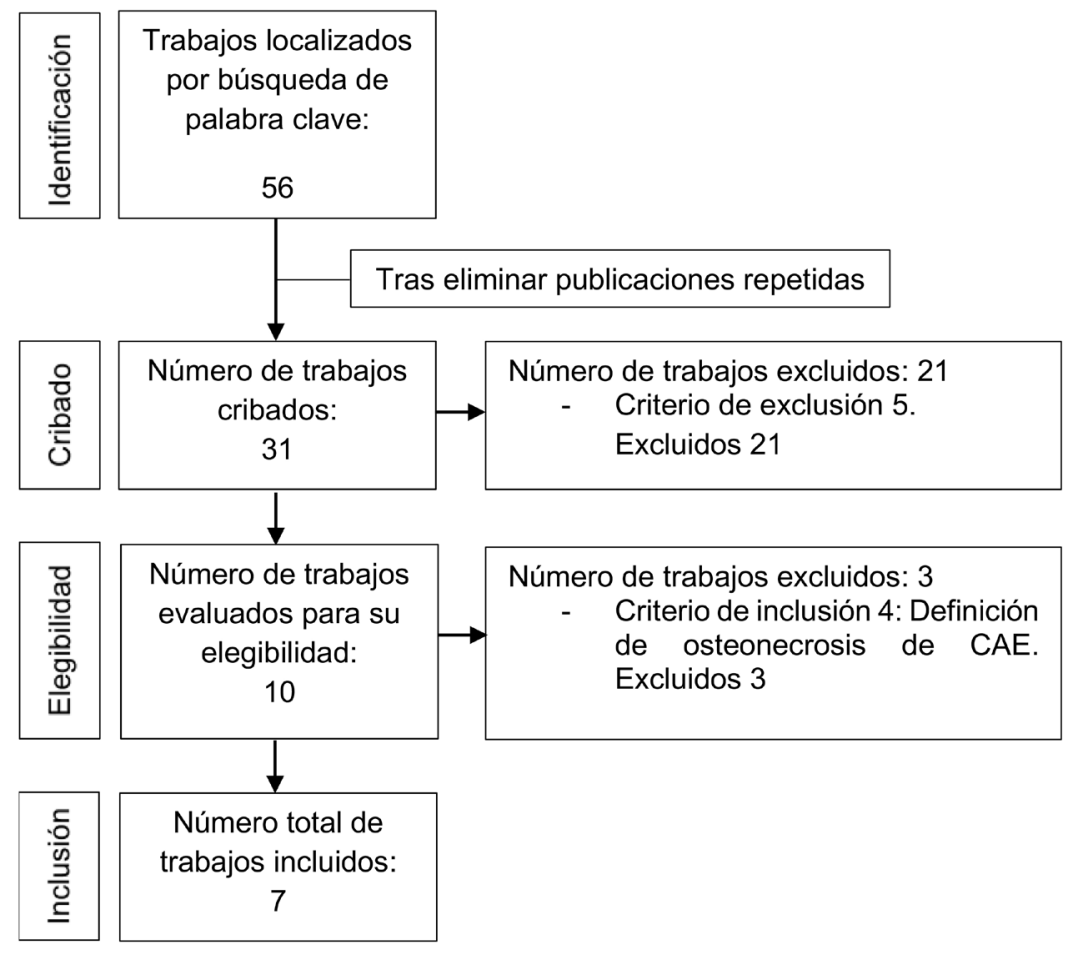

Figura 1. Diagrama de flujo del proceso de revisión sistemática.

\section{RESULTADOS}

Se incluyeron un total de siete artículos que cumplían los criterios de inclusión propuestos y fueron seleccionados para la revisión sistemática. Tras la lectura de los diferentes resúmenes publicados, se excluyeron los artículos que no cumplían los criterios y aquellos que aparecieron duplicados. La búsqueda en MEDLINE vía PubMed, Web of Science (WOS) y la Biblioteca Cochrane dio un total de diez resultados. De estos, tres artículos fueron descartados tras la lectura de los resúmenes y ver que no cumplían los criterios de inclusión definidos a priori. El texto completo de los siete artículos restantes fue examinado en detalle. Un resumen de los principales resultados está reflejado en la Tabla 1 adjunta organizados de forma cronológica de la publicación más antigua a la más reciente.

Los siete trabajos publicados, lo fueron entre los años 2006 a 2018, ambos incluidos, en seis países de dos continentes diferentes (Europa y Oceanía). El número de casos descritos en cada uno varió desde uno hasta seis en McCadden et al. No se encontró ningún estudio relevante no publicado.

En total, la revisión incluye 17 pacientes mayores de 18 años con diagnóstico confirmado de osteonecrosis del conducto auditivo externo en el 100\% según la definición descrita por la AAOMS. El $76 \%$ fueron mujeres y el $24 \%$ restante varones, con edades comprendidas entre 51 y 89 años. La 


\section{OSTEONECROSIS DE CONDUCTO AUDITIVO EXTERNO SECUNDARIA A BIFOSFONATOS. REVISIÓN SISTEMÁTICA DE LA LITERATURA}

LÓPEZ-SIMÓN E ET AL

distribución global, teniendo en cuenta el uso de los distintos bifosfonatos, fue de cinco pacientes tomando alendronato $70 \mathrm{mg}$ semanales por vía oral $(29,41 \%)$, tres recibieron la combinación de pamidronato $90 \mathrm{mg}$ y zoledronato $4 \mathrm{mg}$ mensuales por vía intravenosa (17,64\%), uno de los cuales asoció, además, dexametasona $40 \mathrm{mg}$ diarios durante cuatro días al mes. Un paciente recibió pamidronato $90 \mathrm{mg}$ en monoterapia de forma mensual por vía intravenosa $(0,058 \%)$, zoledronato $4 \mathrm{mg}$ cada 3 meses por vía intravenosa $(0,058 \%)$, clodronato oral $1600 \mathrm{mg}$ diarios de forma discontinua (0,058\%). En el artículo de McCadden et al, no se especificó el tipo de bifosfonato administrado en ninguno de los seis casos descritos (35,29\%). La duración del tratamiento y tiempo hasta la aparición de sintomatología fue desde los veinte meses hasta los quince años en alguno de los pacientes.

Las siguientes enfermedades de base fueron registradas en los pacientes revisados: once sujetos con diagnóstico de osteoporosis $(64,70 \%)$, tres con mieloma múltiple IgG kappa con trasplante autólogo de médula ósea (17,64\%), dos con cáncer de mama que recibieron tratamiento de quimiorradioterapia (excluyendo la región craneocervical) $(11,76 \%)$ y uno plasmocitoma con trasplante autólogo de médula ósea $(0,05 \%)$.

La forma más habitual de presentación fue la úlcera sobreinfectada con exposición ósea unilateral en siete pacientes $(41,17 \%)$, bilateral en seis pacientes $(35,29 \%)$ y sin especificar lateralidad en los cuatro restantes $(23,52 \%)$. La zona de aparición inicial fue el suelo del CAE en todos los pacientes. En cuanto a la sintomatología acompañante, seis casos $(35,29 \%)$ acudieron a consulta por el comienzo o exacerbación de un tapón de cera, y los once restantes $(64,71 \%)$ relataban clínica infecciosa de otitis externa crónica (otalgia, otorrea). Además, en ocho pacientes (47,05\%) se constató la presencia de exposición ósea uni o bilateral. En cuanto a la presencia de sobreinfección, diez pacientes $(58,82 \%)$ tuvieron datos a favor de esta.
Como pruebas de imagen diagnósticas complementarias, se realizó tomografía axial computarizada (TC) a ocho de los casos, observando signos de necrosis y de secuestro óseo; y resonancia magnética (RM) a un paciente con resultado dentro de la normalidad. Se biopsió la zona ulcerada del CAE a dos de los pacientes dando signos de osteomielitis como resultado anatomopatológico.

En relación con el tratamiento empleado, cinco pacientes $(29,41 \%)$ fueron tratados con antibiótico y corticoide tópico, observando signos de recurrencia únicamente en uno de ellos. En cuatro $(23,52 \%)$ se emplearon antibióticos tópicos en monoterapia, apareciendo recurrencia en el 50\%. Los ocho pacientes restantes (47,05\%) precisaron, además, técnicas quirúrgicas de desbridamiento y reconstrucción con fascia del músculo temporal, pericondrio o cartílago. Se constató recurrencia en dos de estos últimos pacientes quirúrgicos a los seis y doce meses, respectivamente. El cese del tratamiento con BFS no se realizó de forma estandarizada y cada paciente recibió un enfoque distinto según sus características y variables individuales.

\section{DISCUSIÓN}

La primera publicación de los efectos biológicos de los difosfonatos, posteriormente renombrados como bifosfonatos (BFS), data del año 1969. Es bien sabido que actúan inhibiendo la resorción ósea interfiriendo en la acción de los osteoclastos. Todos los BFS usados actualmente como tratamiento médico poseen dos enlaces P-C unidos por un carbono y son químicamente análogos estables de compuestos de pirofosfato. Como grupo, comparten propiedades y características, sin embargo, existen diferencias químicas, bioquímicas y farmacológicas evidentes entre todos ellos [4]. Se clasifican principalmente atendiendo a la vía de administración. Los BFS por vía intravenosa tienen 10,000 veces más efectos secundarios que por vía oral. En contraste, algunos autores sugieren que el contenido de nitrógeno de los BFS puede 


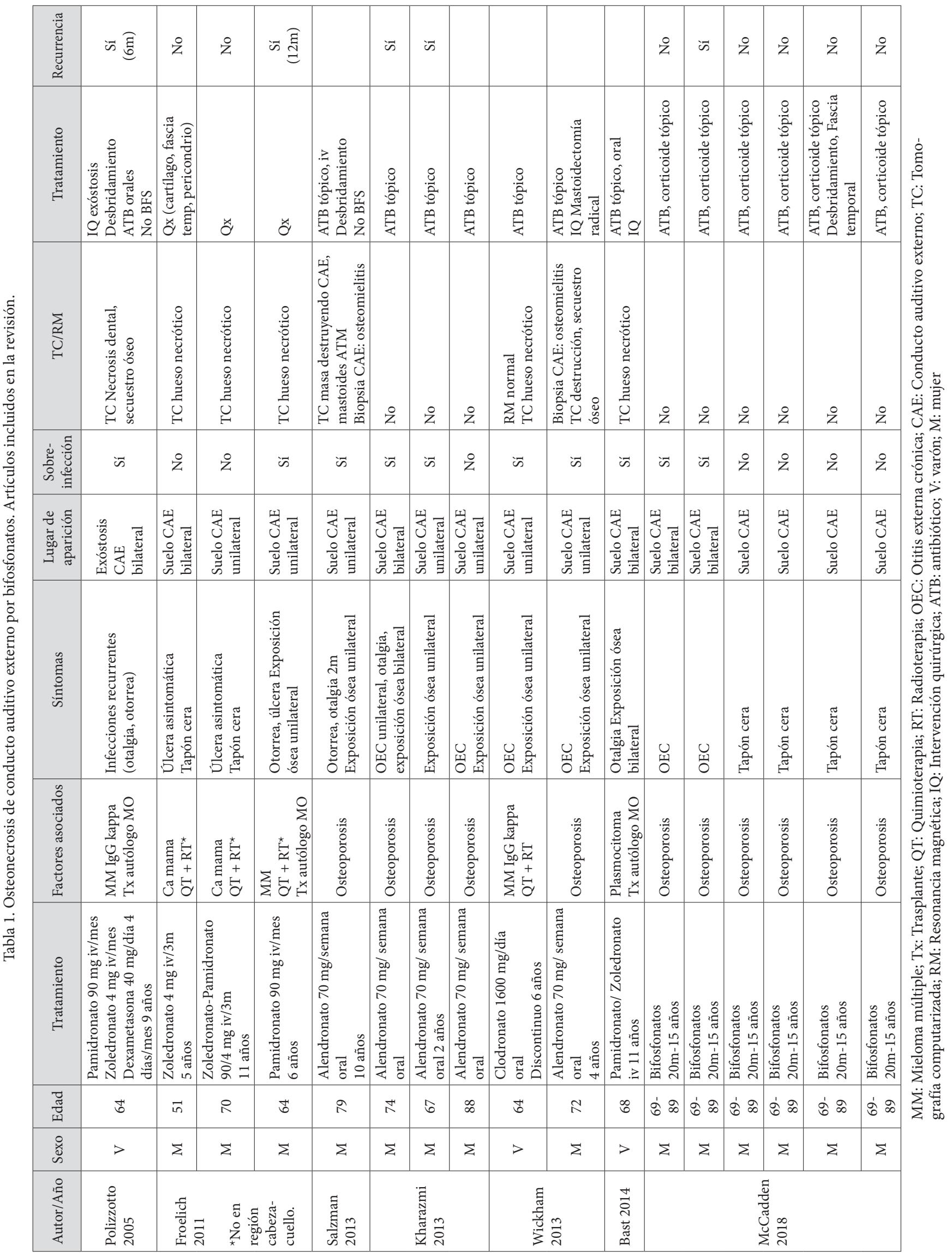




\section{OSTEONECROSIS DE CONDUCTO AUDITIVO EXTERNO SECUNDARIA A BIFOSFONATOS. REVISIÓN SISTEMÁTICA DE LA LITERATURA}

ser un factor de riesgo más importante incluso que la forma de administración. Los estudios más recientes, dividen los BFS en dos clases: nitrogenados (tanto orales como iv) y no-nitrogenados (solo orales) $[8,9]$. Son empleados como parte del tratamiento de la enfermedad de Paget, hipercalcemia tumoral, mieloma múltiple y metástasis óseas, entre otras.

La osteoporosis y el bajo índice de masa ósea son un problema frecuente que afecta principalmente a la población femenina. Se estima que el $50 \%$ de las mujeres y $20 \%$ de hombres de más de 50 años tienen riesgo de sufrir una fractura osteoporótica $[2,11]$. Los BFS son el primer tratamiento de elección en la mayoría de las pacientes con osteoporosis. Los efectos secundarios frecuentes relacionados con su uso son bien conocidos pero pueden surgir potenciales complicaciones que tenemos que sospechar [14]. En el año 2003, Marx describió el primer caso de osteonecrosis de mandíbula asociado al tratamiento con BFS, pero no fue hasta el 2007 cuando la Asociación Americana para la Investigación del Hueso y del Mineral (ASBMR) lo definió $[6,8]$. Este hecho nos lleva a pensar que es necesario tratar a numerosos pacientes para llegar a descubrir efectos secundarios raros del tratamiento. Aunque la fisiopatología de la enfermedad no está completamente elucidada, se piensa que intervienen mecanismos como la inhibición de los osteoclastos en la resorción y remodelación de hueso, fenómenos de inflamación local e infección o la inhibición de la angiogénesis [8]. El desarrollo de osteonecrosis mandibular está en relación con la potencia del BFS empleado [5]. Existen muy pocos casos publicados de osteonecrosis mandibular en pacientes consumidores de BFS no-nitrogenados en comparación con los más de 3000 casos publicados de los BFS nitrogenados como el alendronato, pamidronato y zoledronato $[5,9,10]$.

La osteonecrosis benigna del conducto auditivo externo (CAE) es un diagnóstico clínico poco conocido entre especialistas de otorrinolaringología, pudiendo incluso confundirse con otra enfermedad, como el colesteatoma según refiere Thorsteinsson et al. En el año 2006, Polizzoto et al [7] fue el primero en describirlo en un paciente con mieloma múltiple tratado con BFS intravenosos y desde entonces, según nuestra revisión, solo hemos recopilado un total de 17 casos publicados a nivel mundial. Todos presentaron osteonecrosis del CAE tras el consumo de BFS, 6 de ellos de forma oral y 5 por vía intravenosa, durante un periodo de tiempo prolongado (Tabla 1). Se caracteriza por la aparición de una zona ulcerada circunscrita e indolora en el suelo del CAE [10]. Algunos pacientes pueden presentar otalgia o infecciones de repetición [13].

No existen factores de riesgo descritos para el desarrollo de la osteonecrosis del conducto [7]. Salzman et al, hablan del impacto del tapón de cera con formación de herida en el CAE como posible factor de riesgo por la colonización bacteriana posterior, así como también, los pequeños traumatismos provocados con los bastoncillos de algodón o los dedos. Un traumatismo repetido en el suelo del conducto puede actuar como factor etiológico de osteonecrosis debido al compromiso de la vascularización, reducción de la viabilidad de los queratinocitos o de las proteínas de la matriz extracelular [10]. Por el contrario, se han descrito casos espontáneos sin traumatismo previo y probablemente no sea necesario para el desarrollo de osteonecrosis del canal [8]. La patogenia de la enfermedad es aún desconocida, aunque se han sugerido numerosas posibles explicaciones [9]. Debe distinguirse de la radio-osteonecrosis o de la presencia de carcinoma.

Los pacientes consumidores de BFS tienen un riesgo significativamente elevado de desarrollar osteonecrosis del CAE. Este hecho aumenta cuanto más duradero y mayor sea la dosis del tratamiento. Sin embargo, dada la baja incidencia de estos dos posibles efectos secundarios y el demostrado beneficio del uso de BFS en pacientes osteoporóticos con alto riesgo de fractura, la balanza se inclina hacia su prescripción $[1,12,14]$. 


\section{OSTEONECROSIS DE CONDUCTO AUDITIVO EXTERNO SECUNDARIA A BIFOSFONATOS. REVISIÓN SISTEMÁTICA DE LA LITERATURA \\ LÓPEZ-SIMÓN E ET AL}

De acuerdo con la literatura, los casos publicados corresponden en su mayoría a pacientes con diagnóstico de osteoporosis (64,70\%), y en menor frecuencia con patología maligna $(23,52 \%)$, sometidos a quimiorradioterapia previa de áreas fuera de la región cervicofacial. Las técnicas de imagen no son en principio necesarias para establecer el diagnóstico. El TAC de hueso temporal es de utilidad para determinar la extensión, excluir la presencia de mieloma subyacente y descartar metástasis óseas. La gammagrafía ósea con Tecnecio-99 puede ser de utilidad ya que es capaz de detectar osteonecrosis subclínica, confirmar el diagnóstico de osteomielitis y distinguirlo de malignidad [9]. Sin embargo, esta prueba no se realizó en ningún caso.

La osteonecrosis en relación con el uso de BFS debe ser parte del diagnóstico diferencial de las lesiones del hueso temporal en el que debemos tener en cuenta la otitis externa maligna, osteoradionecrosis del hueso temporal, colesteatoma del conducto auditivo externo y formación de metástasis óseas primarias o secundarias [9].

No existe claro consenso entre el cese o no de los BFS, por lo que es importante evaluar de forma individual cada caso. La ASBMR recomienda el uso de antibióticos tópicos y reserva la forma sistémica para los casos agudos. En cuanto a las técnicas quirúrgicas empleadas, el desbridamiento ha dado resultados de efectividad variable y deberían estar limitadas a la eliminación de secuestros óseos y bordes puntiagudos que puedan provocar traumatismo directo repetido en el CAE [6].

A pesar de ser un efecto adverso infrecuente, la osteonecrosis del CAE debe sospecharse en aquellos pacientes que estén tomando o hayan tomado BFS por la significativa morbilidad que conlleva [11].

\section{CONCLUSIONES}

La osteonecrosis del conducto auditivo externo es un efecto secundario muy infrecuente del consumo de bifosfonatos. Como especialistas, debemos estar alerta de su posible aparición en pacientes tratados durante largo tiempo y sospecharlo si presenta síntomas de patología en el conducto auditivo externo.

Es importante realizar una completa historia clínica haciendo hincapié en el tipo, dosis y duración del tratamiento previo. Aunque las pruebas de imagen no son imprescindibles para el diagnóstico, la tomografía computarizada y la gammagrafía ósea pueden ser de utilidad en los casos de osteonecrosis más extensos.

El tratamiento consiste habitualmente en la administración de antibióticos \pm corticoides tópicos. En un menor porcentaje de casos, es preciso realizar técnicas quirúrgicas para su correcta resolución.

\section{BIBLIOGRAFÍA}

1. Thorsteinsson AL1, Vestergaard P, Eiken P. External auditory canal and middle ear cholesteatoma and osteonecrosis in bisphosphonate-treated osteoporosis patients: a Danish national register-based cohort study and literature review. Osteoporos Int 2014;25:1937-44.

2. Kavanagh KL, Guo K, Dunford JE, Wu X, Knapp $\mathrm{S}$, Ebetino FH. The molecular mechanism of nitrogen-containing bisphosphonates as antiosteoporosis drugs. Proc Natl Acad Sci USA 2006;103:7829-34.

3. Fleisch H, Russell RG, Straumann F. Effect of pyrophosphate on hydroxyapatite and its implications in calcium homeostasis. Nature 1966;212:901-3.

4. Russell RG. Bisphosphonates: the first 40 years. Bone 2011;49:2-19.

5. Wickham N, Crawford A, Carney AS, Goss AN. Bisphosphonate-associated osteonecrosis of the external auditory canal. J Laryngol Otol 2013 Jul;127 Suppl 2:S51-3.

6. Khosla S, Burr D, Cauley J, Dempster DW, Ebeling PR, Felsenberg D. Bisphosphonate-associated osteonecrosis of the jaw: report of a task force of the American Society for Bone and Mineral Research. J Bone Miner Res 2007;22:1479-91. 


\section{OSTEONECROSIS DE CONDUCTO AUDITIVO EXTERNO SECUNDARIA A BIFOSFONATOS. REVISIÓN SISTEMÁTICA DE LA LITERATURA LÓPEZ-SIMÓN E ET AL}

7. Polizzotto M, Cousins V, Schwarer A. Bisphosphonate-associated osteonecrosis of the auditory canal. Br J Haematol 2006;132:114.

8. Ruggiero SL, Dodson TB, Fantasia J, Goodday R, Aghaloo T, Mehrotra B. American Association of Oral and Maxillofacial Surgeons position paper on medication-related osteonecrosis of the jaw--2014 update. J Oral Maxillofac Surg 2014;72:1938-56.

9. Salzman R, Hoza J, Perina V, Starek I. Osteonecrosis of the external auditory canal associated with oral bisphosphonate therapy: case report and literature review. Otol Neurotol 2013; 34:209-13.

10. Froelich K, Radeloff A, Kohler C, Mlynski R, Muller J, Hagen R et al. Bisphosphonate-induced osteonecrosis of the external ear canal: a retrospective study. Eur Arch Otolaryngol 2011; 268:1219-25.

11. McCadden L, Leonard CG, Primrose WJ. Bisphosphonate-induced osteonecrosis of the ear canal: our experience and a review of the literature. Jour Otol Laryngol 2018;1-3.
12. Bisphosphonates: osteonecrosis of the external auditory canal. Prescrire Int $2016 ; 25: 154$.

13. Kharazmi M1, Hallberg P, Persson U, Warfvinge G. Bisphosphonate-associated osteonecrosis of the auditory canal. Br J Oral Maxillofac Surg 2013;51:e285-7.

14. McClung M, Harris ST, Miller PD, Bauer DC, Davison KS, Dian L et al. Bisphosphonate therapy for osteoporosis: benefits, risks, and drug holiday. Am J Med 126:13-20.

15. Bast F1, Fuss H, Schrom T. Bilateral bisphosphonate-associated osteonecrosis of the external ear canal: a rare case. HNO 2012;60:1127-9.

16. Liberati A, Altman DG, Tetzlaff J, Mulrow C, Gotzsche PC, Ioannidis JP. The PRISMA statement for reporting systematic reviews and meta-analyses of studies that evaluate health care interventions: explanation and elaboration. J Clin Epidemiol 2009;62:e1-34. 
